Escuela de Ciencias Sociales y Humanidades, UNED, C.R. URL: http://investiga.uned.ac.cr/revistas/index.php/espiga/index

\title{
Los peces de Cooper y el género detectivesco: del dominante epistemológico al dominante ontológico
}

\section{Roy Alfaro-Vargas}

Bachiller en la Enseñanza del Castellano y la Literatura, y egresado del Posgrado en Literatura Latinoamericana (Universidad de Costa Rica). Ha sido profesor en la Universidad de Costa Rica y en el Instituto Tecnológico de Costa Rica. Asimismo, es investigador independiente. Además, cuenta con traducciones publicadas del francés, inglés y alemán al español. Investigador independiente. Costa Rica.royalfarov@gmail.com

Recibido: 06 de abril, 2016 - Aceptado: 10 de mayo, 2016 - Corregido: 18 de agosto, 2016

\section{RESUMEN}

(Find the abstracts in English and French at the end of the article)

Este artículo analiza Los peces de Cooper en relación con los parámetros del género detectivesco clásico y del género detectivesco metafísico, el primero ligado a las poéticas modernistas y el segundo a las postmodernistas. El análisis plantea un choque entre ambas poéticas, a través de la incorporación del elemento ficto-científico de la superconductividad. Asimismo, se interpreta esta novela (Los peces de Cooper) como una literatura experimental anclada en las narrativas innaturales.

Palabras clave: literatura costarricense, género detectivesco, física, narrativas innaturales, literatura experimental.

\section{Introducción}

La novela Los peces de Cooper $(L P C)$ de Manuel Ortega-Rodríguez y Alí VíquezJiménez, es un relato que nos plantea ya, desde la misma idea de la superconductividad y de la complejidad, un debate de extrema actualidad, al no solo referirnos a cuestiones como el "fin" del positivismo, la mecánica cuántica, etc., sino también a la relación entre

\section{Formato de citación según APA}

Alfaro-Vargas, R. (2016). Los peces de Cooper y el género detectivesco: del dominante epistemológico al dominante ontológico. Revista Espiga. Vol. XV, (32), 153-167.

\section{Formato de citación según Chicago}

Alfaro-Vargas, Roy. «Los peces de Cooper y el género detectivesco: del dominante epistemológico al dominante ontológico». Revista Espiga. XV. n 32 (2016): 153-167. 
ciencia y literatura, muy discutida últimamente en función de lo que se ha denominado ciencia ficción, en Costa Rica ${ }^{1}$.

Asimismo, $L P C$ se estructura como una novela detectivesca, lo cual produce algunas peculiaridades al coexistir con los lineamientos de la teoría de la complejidad, en tanto elemento ficto-científico (como se verá más adelante).

El argumento es el siguiente: $L P C$ se define por el cruce onto-epistemológico de algunos elementos pertenecientes a las poéticas modernistas (el dominante epistemológico de la noción clásica del género detectivesco) y algunos elementos asimilados por la postmodernidad (como la teoría de la complejidad y algunas interpretaciones filosóficas de la mecánica cuántica, así como en relación con las narrativas innaturales), en el marco del desarrollo de un factor ficto-científico, como lo es la elaboración de superconductores a temperatura ambiente.

En procura de dar fundamento al anterior argumento, es preciso antes definir el género detectivesco y determinar la relación de este, por un lado, con lo moderno y, por otro lado, con lo postmoderno, sin olvidar plantear qué es la superconductividad, en relación con la mecánica cuántica y la teoría de la complejidad.

\section{El género detectivesco}

Dicho de manera expresa, "(...) la ficción detectivesca refiere a una narrativa cuya principal acción es acerca del intento de un investigador por resolver un crimen y llevar al criminal ante la justicia" (Pyrhönen, 2010, p. 43)². El género detectivesco se establece entonces como una narración donde hay una relación causa/efecto, que se hace evidente a través de la evidencia empírica. En este sentido, es que se liga este género a las poéticas modernistas, mediante la idea de un dominante epistemológico (McHale, 1987).

Claramente, en este género, la figura del detective es primordial, en tanto este "(...) es una figura cuyo trabajo es decodificar algunos signos e imponer un orden narrativo a una masa aparentemente caótica de detalles" (Nicol, 2009, p. 172). En esta perspectiva, el detective marcará el tiempo de la investigación (en tiempo presente) de la historia, estructurando el who is guilty?, el ¿quién es culpable? (Pyrhönen, 2010).

Sin embargo, dentro de la ficción detectivesca, hay un desplazamiento temporal, que, aparte del tiempo de la investigación en tiempo presente, está fundamentado en el tiempo del relato -lo que en inglés llaman el whodunit (Pyrhönen, 2010).

El whodunit (el relato detectivesco) plantea el crimen, a diferencia del who is guilty?, que muestra un fenómeno de índole más general como lo es la culpa. El whodunit señala así la particularidad de este crimen: "[l]a cuestión del "Whodunit" no es idéntica a la pregunta de "Who is guilty?", porque la investigación muestra que la culpa es un fenómeno

1. Ya el mismo Víquez (2014) ha manifestado algunos de los supuestos sobre la relación ciencia-literatura, alrededor de la idea de los modelos ficcionales del mundo.

2. Todas las traducciones de textos citados en lengua inglesa, son del autor. 
más universal que el crimen" (Pyrhönen, 2010, p. 44). Por tanto, la culpa es parte de un hecho de carácter general, mientras el whodunit es la plasmación particular de la culpa.

La relación entre el tiempo de la investigación y el tiempo del relato, por su parte, inicia con una omisión, es decir, se "(...) omite narrar el momento de cometerse el crimen..." (Pyrhönen, 2010, p. 49). Es decir, en la literatura detectivesca existe una “(...) compleja doble narrativa, en la cual un relato ausente, aquel del crimen, es gradualmente reconstruido en el segundo relato (la investigación)...” (Marcus, 2003, p. 245). Esta diferencia entre el tiempo de la historia y aquel del relato implica que existe la conciencia de estar ante un acto narrativo, artificial, lo cual da un rasgo característico a este tipo de género, en cuanto "(...) este refleja su altamente auto-consciente conciencia (highly self-conscious awareness) de la artificialidad de la narración y de la ambigüedad de los relatos" (Cawelti, 1997, p. 11).

Así entendido, el género detectivesco tiene dos tramas (la del crimen y la de la investigación) amparadas en tiempos divergentes (el pasado para el crimen y el presente para la investigación) los cuales se enlazan en la resolución del crimen. Asimismo, esta estructura tempo-narrativa doble y tergiversante (Cawelti, 1997) puesta en función el whodunit y del who is guilty?, nos coloca de frente ante la cuestión de la relación entre la ilegalidad (el whodunit) y la inmoralidad que pesa sobre la culpa (el who is guilty?), distinguiendo "(...) entre lo correcto y lo incorrecto" (Pyrhönen, 2010, p. 44). Pyrhönen (2010) afirma que:

Este género aborda perennes temas literarios como los conflictos impersonales, la motivación humana y la escogencia moral. La investigación siempre incluye un análisis de la interacción humana conducente al crimen. Con el fin de determinar la identidad del culpable, el detective evalúa cómo la responsabilidad moral es asignada entre los sospechosos. Sus evaluaciones toman en cuenta la diferencia entre los códigos judiciales y morales... (p. 44).

El género detectivesco, en esta perspectiva, aborda la naturaleza humana, ligada a al comportamiento criminal, dentro de una evaluación jurídico-moral. Lógicamente, esta evaluación jurídico-moral se realiza desde una puntual ideología, que aplica sanciones morales y jurídicas, según correspondan. Así, ya desde el siglo XIX, este género literario, como señala Marcus (2003), se inserta en y reproduce

(...) una cultura en la cual el crimen y el castigo están ambos profundamente internalizados en la esfera subjetiva y externalizados en aún más elaboradas formas de disciplina y vigilancia, y en nuevas instituciones legales y procedimientos judiciales, en particular la formación de la policía detectivesca, el surgimiento de las ciencias forenses y el desarrollo de juicios basados en la evidencia (p. 246).

De esta manera, el género detectivesco está conectado con el desarrollo de las ciencias jurídico-criminológicas, así como a la visión de mundo de la sociedad burguesa. En este marco, clásicamente, la ficción detectivesca representa "el crimen, como un problema distintivo de las sociedades burguesas, individualistas y cuasi-democráticas" (Cawelti, 1997, p. 12). Puesto que, por un lado, el crimen supone la existencia de la esfera privada (fenómeno ligado a la concepción filosófico-antropológica de la burguesía) y, por otro lado, según Cawelti (1997): 
(...) el detective nos revela por sus acciones que, a pesar de lo corrupta e injusta que esta sociedad pueda ser en algunos casos particulares, esta todavía contiene la inteligencia y los medios para definir y exorcizar estos males en tanto problemas particulares" (p. 12).

En otras palabras, la sociedad burguesa genera tales males "particulares", mas también procesos racionales y racionalizados (la ciencia forense, el sistema jurídico-legal, etc.) los cuales finalmente legitiman "(...) el poder de los grupos socialmente dominantes..." (Pyrhönen, 2010, p. 47). De hecho, este género “(...) no contesta los estándares habituales y cotidianos de percepción y pensamiento" (Pyrhönen, 2010, p. 48). En efecto, este género se dedica a crear una visión epistemológica (el dominante epistemológico) en el cual lo importante es revelar “(...) las conexiones lógicas, causales y temporales entre los eventos" (Pyrhönen, 2010, p. 50); mientras el trasfondo social del texto se substancializa, ya que no se cuestiona el mismo. Dicho de otro modo, el dominante epistemológico en la ficción detectivesca solo busca encontrar la "verdad" de los hechos particulares narrados, es decir, del crimen, afianzando la racionalidad positivista (por lo menos en la clásica literatura detectivesca).

Sin embargo, dentro del fenómeno postmoderno, el género detectivesco sufre un desplazamiento desde el dominante epistemológico, al ontológico; lo cual introduce algunos cambios con respecto a la versión clásica de este género.

La estética realista del dominante epistemológico (anclada en la relación pasadopresente del whodunit y el who is guilty? y basada en probar una verdad, hablando judicial y moralmente) se transforma dentro del postmodernismo en un dominante ontológico: este cambia de estilo desde las poéticas modernistas del dominante epistemológico a una poética postmodernista del dominante ontológico (McHale, 1987). Dentro del dominante ontológico, ya no es fundamental la verdad, sino la elaboración no-realista (en el contexto anglosajón se preferiría aquí la palabra "metafísica") de una realidad no-empírica y no-correspondiente a ninguna realidad probablemente existente. En lugar de la estructuración pasado-presente del género detectivesco clásico, modernista, se encuentra una literatura detectivesca, con un espectro temporal que incluye el pasado, el presente y, por extensión, el futuro; denominada relato detectivesco metafísico o relato detectivesco postmoderno (Merivale, 2010).

Este relato detectivesco metafísico es metafísico, en tanto va más allá de la realidad del mundo empírico, el cual parece interpretarse como existente en pasado-presente. Para ir más allá de un tal mundo empírico, la literatura detectivesca metafísica entra en un maridaje con la ciencia ficción, en cuanto esta última tiene un elemento futurista: "[e]l relato detectivesco metafísico ha también hallado su camino en las afueras de la ciencia ficción, el género del 'futuro"' (Merivale, 2010, p. 319). Así, el no haber existido y el no-existir hic et nunc del futuro, permite a este relato detectivesco metafísico reforzar "(...) su interés en los mundos confrontantes con una auto-reflexiva preocupación acerca de los mundos literarios" (Nicol, 2009, p. 165) ${ }^{3}$. Este género detectivesco metafísico plantea ontologías alternas, que se insertan en lo que más adelante llamamos las narrativas innaturales.

3. Aquí es válido recordar que "[l]a ciencia ficción, y su potencial para ofrecer una alternativa al realismo -y una crítica de este- ha probado ser fundamental para el postmodernismo" (Nicol, 2009, p. 164). 
Además de incorporarse el futuro como un elemento más dentro del relato detectivesco metafísico, se asume abiertamente la idea del texto como un artificio, o sea, se introduce en la literatura detectivesca un factor metaficcional. De ahí que este género es visto, dentro de la visión postmoderna, “(...) como un género que es naturalmente metaficcional y que nos provoca - como lo hace la ficción postmoderna, en algún sentido- mediar sobre las prácticas de escribir y leer ficción" (Nicol, 2009, p. 183), donde el proceso de escritura-lectura, supondrían los postmodernos, se abre a la libre interpretación, ya que no habría realismo, sino solo realidades alternas.

\section{La superconductividad}

La superconductividad fue descubierta en Leiden, en 1911, por Kamerling Onnes. Este fenómeno consiste en un desafío de "(...) las leyes usuales de la electricidad..." (Blundell, 2009, p. 2), en cuanto en los superconductores está ausente el fenómeno denominado el calentamiento de Joule (Blundell, 2009). Es decir, en un cable eléctrico de uso doméstico, la electricidad sufre un proceso de resistencia, que no solo desperdicia energía eléctrica, sino que también produce un calentamiento. En los superconductores, la ausencia de calentamiento permitiría una circulación eterna de la electricidad (Blundell, 2009).

Por otra parte, es sabido que "[p]ara que la superconductividad ocurra, el material tiene que ser enfriado a una muy baja temperatura" (Blundell, 2009, p. 4), medida en grados Kelvin.

En tanto fenómeno ligado a la mecánica cuántica, en la superconductividad “(...) un enorme número de electrones actuaría en concierto, como si cada uno fuese parte de un más extenso, inseparable todo (whole)" (Blundell, 2009, p. 61). Se produciría, en la superconductividad, un efecto sinérgico, emergente (teoría de la complejidad), donde un número de electrones, por ejemplo, presentarían propiedades que "(...) emergen de principios organizacionales más altos" (Blundell, 2009, p. 139) y donde “(...) un sistema complejo es más que la suma de sus partes..." (Castellani y Hafferty, 2009, p. 126), lo cual implica que hay un proceso autopoiético que permite al sistema auto-producirse (Castellani y Hafferty, 2009). Esta idea de una sinergia autopoiética es lo que Žižek (2014) conceptualiza como una universalidad no abarcante basada en la división, en partes, en ese nuevo todo (whole) que no es ya una totalidad (totality), lo cual es característico de la particularización que se da en lo postmoderno.

En este marco, la superconductividad es un sistema no-lineal, ya que su funcionamiento subatómico es más que la simple suma de sus partes. De hecho, Cooper en 1956 descubre que un par de electrones (el par de Cooper) en tanto par superconductor tiene “(...) un momento igual y opuesto con respecto a cada otro, aunque el par como un todo puede deslizarse a través de una muestra cuando la corriente fluye" (Blundell, 2009, p. 64). Así, los sistemas no-lineales experimentan comportamientos que no están ligados a sus partes individuales. 


\section{Lo detectivesco en $L P C$}

LPC se estructura en dos partes: la primera parte consta de 16 apartados identificados con números romanos. La segunda parte tiene 10 apartados (también señalados con números romanos) y cinco intercalaciones, cuyos nombres son: Sur, Oeste, Este, Norte y Cenit/Nadir. La primera referencia a leer una intercalación está entre los apartados V y VI, la segunda entre los apartados VI y VII, la tercera entre el VII y VIII, la cuarta entre los apartados VIII y IX, y la última entre el apartado IX y el X.

Esta distribución estructural es importante, en tanto nos remite a algunos procesos formales del género detectivesco, así como a la coexistencia de elementos poéticos modernistas y postmodernistas, en $L P C$.

Los apartados I, II y III de la primera parte refieren a elementos pertinentes a los dos personajes principales: Rebeca y Gregorio (Goyo) Mirarbueno. Así, se muestra, sobre ambos, datos de la niñez, su educación, etc. Realmente, estos primeros apartados no se insertan todavía en el whodunit propiamente dicho. Estos apartados son parte de un proceso formal del género detectivesco llamado ambigüedad, cuya función es impedir que el lector perciba el todo, generando en el lector una serie de múltiples inferencias (Pyrhönen, 2010). Así, como vemos con estos tres primeros apartados, la ambigüedad incluye, en $L P C$, " 'narrativas fantasma' que no están basadas en el relato [en el whodunit] sino que tienen grados variables de existencia en el discurso o en la mente del lector" (Pyrhönen, 2010, p. 50; corchetes míos). Las "narrativas fantasma" representan elementos que o no son pertinentes para la comprensión del whodunit $y$ si lo son aparecen de tal modo que no lo parezcan.

En $L P C$, el carácter ambiguo de estos primeros tres apartados de la primera parte incluso evitan que el lector sospeche de la trama detectivesca que se inicia en el apartado V (primera parte). Asimismo, los apartados XI, XII, XIII y XIV elaboran el elemento ficto-científico de $L P C$, en tanto le permiten al lector, de manera muy acertada y precisa, conocer algunos conceptos relacionados con la superconductividad, la teoría de la complejidad, la noción de caos, etc. Si bien estos tienen que ver con el whodunit (en cuanto están relacionados con el objeto del crimen, o sea el robo de la fórmula para la construcción de un superconductor a temperatura ambiente) lo cierto es que, por la misma precisión (que busca un alto grado de verosimilitud) del abordaje de tales conceptos teóricos, nos aleja del proceso del who is guilty?, particularmente, de las entrevistas a John y Edgardo San Pablo (apartados VIII y IX de la primera parte) las cuales continúan en los apartados XV y XVI, con las entrevistas a los otros dos sospechosos, es decir, a Carolina y Alberto.

En este marco de inserción de los elementos ficto-científicos (apartados del XI al XIV, primera parte) se crea un proceso típico de lo detectivesco, o sea, la fragmentación que rompe el relato de la investigación del crimen, del robo en nuestro caso, en partes, lo cual "(...) permite una recuperación progresiva de eventos pasados y retrasa la comprensión de estos mismos eventos" (Pyrhönen, 2010, p. 50). De hecho, tanto la ambigüedad como la fragmentación señaladas, para $L P C$, producen un patrón de distracción, que “(...) modifica la presentación de los fragmentos...” (Pyrhönen, 2010, p. 50), sumiendo al 
lector en el relato de investigación y desviando la atención del crimen (Pyrhönen, 2010), con lo cual se crea un aire narrativo de mayor intriga y de menor claridad para el lector.

Para comprender esto, es preciso ir al apartado $\mathrm{V}$ de la primera parte, donde inicia el who is guilty?, la historia de la investigación propiamente dicha, y al contexto de este apartado (el apartado IV).

Ya el apartado IV daba el primer indicio de que algo anómalo sucedía, como se ve en el siguiente diálogo entre la tía Mariana y Rebeca: "-A vos te pasa algo, hijita -dijo la tía, aguda- Nunca fuiste buena para disimular. (...)/ -Desembucha. Te descubrimos./ Rebeca se resistió./ -No pasa nada, asuntos de trabajo que van a esclarecerse pronto" (Ortega y Víquez, 2015, p. 16).

En el apartado V, Rebeca ahí sí declara, con un aire muy "cuántico" (o sea, solo como posibilidad azarosa, indeterminada), que: "-Bueno, es posible que alguien haya robado o esté a punto de robar los resultados de nuestro proyecto de investigación" (Ortega y Víquez, 2015, p. 19).

Fiel al fundamento cuántico de la superconductividad (los pares de Cooper), el texto plantea su whodunit en términos de posibilidad y no de hecho consumado (aunque quizás sí sea de ese modo), de realidad en sí. La incertidumbre heisenberguiana se estructura como incertidumbre epistemológica, en cuanto la verdad/falsedad del robo, que formaliza el whodunit, se sale de los dominios del tercero excluido de la lógica formal, el cual implica que A es A o no-A, pero no ambos a la vez, lo cual es un rasgo postmoderno, que ya nos inserta en el choque entre la poética modernista y la postmodernista.

De la misma manera, planteado el crimen, solo queda, dentro de las premisas del género detectivesco seguidas por $L P C$, encontrar al investigador. De hecho, la tía Mariana designa al investigador (con lo cual inicia el who is guilty?) al proponer a Goyo, como tal: "-Pues de que este muchacho ya encontró cómo retribuirte por la mensualidad que le has asignado. Se va a hacer cargo de la investigación criminal que te preocupa, hija" (Ortega y Víquez, 2015, p. 31).

Como se aprecia, el relato de la investigación se monta sobre un hecho "pasado" (por lo menos en lo que respecta al móvil del crimen), el robo de los datos del superconductor, que es el que articula el whodunit. Sin embargo, en $L P C$, el elemento ficto-científico (ligado a lo cuántico) que es el objeto del crimen, también estructura la novela, en tanto las intercalaciones junto a la fragmentación-ambigüedad-distracción del texto mismo, conllevan un efecto que se quiere estocástico, es decir, tendiente al caos y tratando de evitar o confundir el proceso epistemológico que el relato de investigación implica. En otras palabras, los elementos metaficcionales de las intercalaciones (por ejemplo, en el diálogo entre dos personajes, Alberto y Eduardo, en la intercalación "Este", se lee: "-Entonces no ha de gustarte esta novela" (Ortega y Víquez, 2015, p. 158), donde el artificio narrativo da muestra de la conciencia de sí mismo) y lo ficto-científico crean una realidad alterna ${ }^{4}$,

4. En palabras del mismo Edgardo, en la intercalación Este, se lee: “-Creo que esta es una realidad alternativa" (Ortega y Víquez, 2015, p. 157). 
una ontología, donde existen otras posibilidades no-realistas (entendido lo no-realista en términos epistemológicos y, asimismo, estéticos).

De vuelta al ámbito de la investigación, que es el ámbito epistemológico por excelencia donde se busca separar lo verdadero de lo falso, la segunda parte de $L P C$ nos encamina hacia una maraña de acciones dolosas, involucrando diferentes personajes, que en cuanto se van manifestando, nos regalan toda una trama plagada de culpables, a la vez que, entre descubrimientos y confesiones de los mismos personajes, se va develando el secreto de la superconductividad a temperatura ambiente. Prácticamente, la resolución de la fórmula de la superconductividad a temperatura ambiente es paralela al descubrimiento del culpable, del ladrón más cínico de todos: John, el físico canadiense, jefe del grupo de investigación.

En la segunda parte de $L P C$, más allá de las intercalaciones, solo el apartado I no corresponde al tiempo pasado del whodunit, ni al presente del who is guilty?, sino que ofrece una visión futura (con respecto al tiempo de la investigación) donde se puede saber que la cuestión de la superconductividad a temperatura ambiente fue desarrollada con éxito. En función de las características poético-modernistas de la versión clásica del género detectivesco, este apartado I no corresponde al desplazamiento temporal del pasado del whodunit y al presente del who is guilty?, más bien introduce un elemento característico del género detectivesco metafísico (en tanto el futuro no ha sido todavía, no ha tenido existencia, mientras el presente es y el pasado fue) de línea postmodernista.

En el apartado I de la segunda parte de $L P C$, se manifiesta la "hibridación" de lo detectivesco con la ciencia ficción, proceso que implica, no sin tensiones, pasar de lo epistemológico, como énfasis modernista, a lo ontológico, como factor determinantemente postmodernista (más adelante se retomará este aspecto).

Asimismo, la segunda parte, como ya el apartado I lo sugiere, continúa con procesos narrativos de fragmentación, distracción y ambigüedad. De hecho, el apartado II, que prosigue con el who is guilty?, presenta a Rebeca con la confesión de que saboteó la fórmula del superconductor: "He saboteado deliberadamente la producción del superconductor" (Ortega y Víquez, 2015, p. 104). Esta confesión, al igual que la de Alberto revelada en el apartado VII de la misma segunda parte, si bien son manipulaciones de la fórmula como las introducidas malsanamente por Edgardo, John y Carolina, poseen un matiz (moralmente hablando) diferente. Así, en referencia a la acción manipulativa de Rebeca, Alberto señala que: “(...) sus intenciones eran buenas al hacerlo” (Ortega y Víquez, 2015, p. 131) y estas intenciones consistían en proteger el proyecto contra algún posible robo, lo cual marca la diferencia de matices entre las acciones de Alberto y Rebeca, con respecto a las de Edgardo, John y Carolina (lo cual también se evidencia en los castigos que se reciben por parte de cada uno de ellos; contrariamente a lo que sucede con Alberto y Rebeca).

En otro orden de cosas, los apartados III, IV y V de la segunda parte, continúan con la historia de la investigación y muestran a Edgardo (físico computacional y seguidor del libre mercado) en contubernio con Carolina y el novio de esta, para robar la fórmula del nuevo superconductor y venderla. Sin embargo, Edgardo traiciona a ambos, escapa al extranjero y vende la fórmula a MySner Inc., sin percatarse que también Carolina había 
introducido un error en tal fórmula, lo cual la hace inservible. Finalmente, Edgardo es acusado y detenido por fraude, quedando a merced del sistema legal.

En el contexto de la traición de Edgardo y Carolina, esta última queda en manos de los deseos de John, quien sumamente enojado, obliga a Carolina a firmar una confesión, como un medio de mantenerla extorsionada, lo cual ejemplifica la función del castigo moral, dentro de $L P C$.

Alrededor de la investigación de Goyo, se dan algunas situaciones que señalan que los errores introducidos separadamente y como producto de la existente desconfianza mutua, por Edgardo y Carolina, se cancelan entre sí (apartado VI, segunda parte), lo cual permite continuar con la producción del superconductor a temperatura ambiente, y que acarreará, en su desarrollo, hallar al mayor traidor.

En el apartado VII, segunda parte, como parte del proceso de fragmentación y distracción del género detectivesco, recordemos que tanto Rebeca, como Alberto, han introducido errores en la fórmula, con el fin de protegerla contra un posible robo. Ante esta situación, John reacciona con gran enojo y despide a Rebeca y Alberto, para luego salir de donde se encontraba furioso en su auto. La indignación de John lleva al lector a no pensar una posible culpabilidad del investigador canadiense.

Sin embargo, hacia el apartado VIII de la segunda parte, sabemos que John tuvo un accidente y que ha caído en coma (castigo moral por su falta) y Carolina escapa y desaparece (una forma de castigo moral, ya que pierde prácticamente su vida). En este contexto, Alberto y Rebeca se lanzan a la búsqueda de la información que John guardó en su computadora (apartados VIII y IX, segunda parte) y descubren que John tenía tratos con i-Cond para vender la fórmula y que "(...) iba a finiquitar una transacción apenas unas horas después de que sufrió el accidente" (Ortega y Víquez, 2015, p. 143). De este modo, cae sobre John una sentencia moral: "Siempre hay que cuidarse de los que predican demasiado la ética” (Ortega y Víquez, 2015, p. 143).

Así, el crimen se resuelve al establecer que Edgardo, Carolina y John habían traicionado por dinero (conducta moral incorrecta) al laboratorio y a la universidad, lo cual trajo para ellos consecuencias de tipo más jurídico-legal para Edgardo y de tipo moral para Carolina y John (para este último en la forma de un castigo casi divino, como lo es el quedar muerto en vida, en coma).

Pyrhönen (2010) afirma que el género detectivesco clásico diferencia entre la culpa moral y la legal, distinguiendo "(...) entre lo correcto y lo incorrecto" (Pyrhönen, 2010, p. 44). En este marco, $L P C$ no se alejan de este requerimiento ideológico moderno o modernista, pero no sin algunas tensiones que se analizarán.

$L P C$ señala una moral y una legalidad individualistas, como también colectivas. Por ejemplo, John y Carolina "reciben" por sus actos erróneos, incorrectos, sanciones de tipo moral: John queda en coma y Carolina huye dejando toda su vida atrás, sin poder regresar. Sin embargo, Edgardo sí recibe un castigo legal doble. Por un lado, es acusado de fraude al vender a MySner Inc. datos falsos y, por otro lado, la esposa presenta contra él una demanda de divorcio. Por ende, a nivel de los personajes, LPC individualiza el castigo que cada uno recibe de acuerdo con su crimen. Edgardo, a quien le cae todo el peso 
de la ley, es el único que hizo mofa de sus compañeros (a través de correos electrónicos): doble culpa, ya que roba y se vanagloria de su crimen. Al parecer, en el plano individual, $L P C$ plantea que cada quien recibe el castigo que merece, según sus actos.

No obstante, en el campo colectivo, social, el asunto es diferente. En este ámbito, parece que el dinero, el poder económico, está por encima de la moral y que lo legal no es necesariamente moral.

De este modo, si bien "La patente de la fórmula de superconductores a temperatura ambiente quedó en nuestra universidad" (Ortega y Víquez, 2015, p. 99), lo cierto es que “(...) el poder de una patente no es más fuerte que el del equipo legal contratado para mantenerla" (Ortega y Víquez, 2015, p. 99), por lo que “(...) es muy improbable que nuestra universidad quede en la historia como el lugar donde se descubrió la superconductividad, e incluso mantenga alguna patente simbólica, pero sin usufructuar de los verdaderos beneficios económicos" (Ortega y Víquez, 2015, p. 100).

De esta manera, lo moralmente correcto sería que la universidad saque provecho económico de su descubrimiento (la superconductividad a temperatura ambiente) pero legalmente es inviable, debido a que el capital transnacional puede pagar una aplicación de la ley pronta y cumplida, apoderándose de la patente. Este elemento de superioridad de lo económico sobre lo legal y lo moral, en $L P C$, nos deja ante un panorama donde la expansión del capital (en nuestra era neoliberal) somete a las demás áreas del Ser social y que, por esta misma condición, está alejado y absolutamente separado de lo moraljudicial. En este sentido, $L P C$ quiere ofrecer una crítica de la dinámica del capital en la era neoliberal, pero, cuando los personajes individuales son castigados, no hay suficiente claridad, ideológicamente hablando, de que las acciones de John, Carolina y Edgardo, son producto de la misma dinámica egoísta, explotadora, lucrativa y oportunista que funciona también a nivel colectivo (corporativamente hablando). John, Carolina y Edgardo actúan de manera moralmente errónea, porque el sistema exige codicia y afán de lucro, cuestión que $L P C$ evade representar y discutir.

Por otra parte, estos códigos de lo moral y legalmente correcto ligados a lo que los postmodernos llaman modernidad, también crean en $L P C$, junto con la necesidad de esclarecer la verdad sobre el robo de la fórmula de los novedosos superconductores, una concepción ontológica que llamaremos realista, en tanto $L P C$ “(...) ofrece ella misma a los lectores como una reflexión del mundo, su transparencia permaneciendo en los modelos familiares de causa y efecto, de relaciones sociales y valores morales. Reproduciendo lo que la sociedad toma por realidad..." (Pyrhönen, 2010, p. 48). Mas, algunos elementos ligados a la superconductividad (su ligamen con la mecánica cuántica), a la cuestión ficto-científica que incorpora en el género detectivesco el tiempo futuro y la metaficcionalidad de las intercalaciones (pensadas también en términos de una lógica cuántica, estocástica) nos plantean el problema de una ontología alterna a la dada por los elementos clásico-detectivescos en $L P C$.

\section{Lo ontológico y lo epistemológico en LPC}

En $L P C$, se encuentra, a nivel temporal, un tiempo pasado relacionado con el whodunit y un tiempo presente insertado en el who is guilty? (el tiempo de la investigación). 
Dentro de estos parámetros, $L P C$ coincidiría con el género detectivesco clásico, donde no solo la cuestión epistemológica es fundamental (encontrar la verdad con respecto al crimen cometido) sino que también se podría interpretar como una manifestación moderno-ontológica basada en una concepción del Ser como empiricidad, en cuanto el pasado y el presente tienen carácter óntico. Es decir, el pasado, aunque ya no existente, fue, como cosa ya existió; y el presente está siendo.

En este orden de cosas, el futuro (dentro del género detectivesco) no sería parte de lo clásico-detectivesco, sino que su incorporación dentro de la trama detectivesca marca el paso de la versión clásica de este género, a lo que Merivale (2010) denomina el relato detectivesco metafísico (o relato detectivesco postmoderno), el cual encuentra, como ya dijimos, "(...) su camino en las afueras de la ciencia ficción, el género del "futuro"” (Merivale, 2010, p. 319). $L P C$ se asume en función de la idea de algo hoy no-existente, o sea, la superconductividad a temperatura ambiente.

De este modo, la relación futuro-ciencia ficción que se incorpora a lo detectivesco es vista como metafísica, en tanto, como ya se apuntó antes, el pasado y el presente han tenido una manifestación empírica, óntica; mientras el futuro, no. En la medida que el futuro no ha existido, este está más allá de la existencia física, luego sería, según tal propuesta, metafísico.

Esta metafisicidad del relato detectivesco postmoderno no es parte en sí del whodunit, ni tampoco del who is guilty?, pero tampoco de la ontología planteada en el relato detectivesco clásico (por estar en un más allá). Por ende, esta metafisicidad no se puede ver en términos de los requerimientos epistemológicos que buscan la verdad del whodunit establecido, en $L P C$. Por consiguiente, el acento se desvía de lo epistemológico (el primer pez de $L P C$ ) a lo ontológico (el segundo pez de $L P C$ ). En este marco, $L P C$ “(...) cruza de la poética modernista del dominante epistemológico a una poética del dominante ontológico" (McHale, 1987, p. 20), donde el elemento ficto-científico (la superconductividad a temperatura ambiente) crea una realidad alterna donde existen características ontológicas que posibilitan tal fenómeno superconductivo que en nuestra realidad es imposible. Lo cual es un "(...) gesto de pura improvisación ontológica...” (McHale, 1987, p. 20), donde "[e]l fundamento es (...) la categoría misma de mundo" (McHale, 2012, p. 146), es decir, donde lo que da sustento es la necesidad estético-literaria de plantear un mundo posible alterno.

Dentro de esta perspectiva y en el contexto de la literatura costarricense, $L P C$ se levanta como una literatura experimental, en tanto, a través del dominante ontológico y de las intercalaciones presentes en esta novela, esta novela establece una narrativa innatural, que "(...) desafía o completamente viola las comprensiones ordinarias de las narrativas y de las voces ficticias y la narración" (Alber, Nielsen y Richardson, 2012, p. 351). De hecho, las narrativas innaturales asumen la elaboración de dominantes ontológicos que presentan metaficcionalmente algún tipo de "(...) motivación anti-mimética, contra-realista o escéptica" (Berry, 2012, p. 129).

Las narrativas innaturales elaboran diégesis con mundos alternos, que “(...) trascienden las posibilidades del mundo real, proyectando escenarios y actos de narración imposibles física, lógica o humanamente" (Alber, Nielsen y Richardson, 2012, p. 351). 
Lo innatural en narratología, en consecuencia, corresponde no solo a la forma (al relato) sino también a la historia. De tal modo, estas diégesis se definen no solo por elaborar ontologías alternas, sino que también pueden implicar nuevas formas de narración (narración en primera persona en tiempo presente, narración paraléptica, etc.) (Alber, Nielsen y Richardson, 2012). Además, la violación de los principios lógico-aristotélicos, como la abolición del principio de tercero excluido, da como resultado la supuesta validación de ontologías alternas donde se pretende que “(...) $p$ y no- $p$ son simultáneamente verdaderos..." (Alber, Iversen, Nielsen y Richardson, 2013, p. 105), que es "[e]1 tipo más obvio de imposibilidad lógica..." (Ryan, 2012, p. 369). En el mundo representado en $L P C$, tan cercano al mundo existente (en términos físicos) de los autores de esta novela, se presenta una condición que no es real en nuestra realidad física y que solo podría existir en una muy lejanamente posible realidad alterna, con lo que $p$ (los principios físicos de nuestra realidad) coexistiría a la vez con un elemento que la niega (o sea, la superconductividad a temperatura ambiente, por ende no-p): imposibilidad física y lógica.

Por tanto, lo narrativo innatural en $L P C$ se observa en el dominante ontológico del elemento ficto-científico de la superconductividad a temperatura ambiente y, en el ámbito narratológico, en las intercalaciones. El dominante ontológico en $L P C$ permite representar otro mundo donde el fenómeno de la superconductividad a temperatura ambiente es posible (o sea, un espacio ontológico alterno), pero imposible (hasta hoy) en nuestro mundo real. Así, en $L P C$, se puede “(...) observar un proceso de mediación dialéctica entre componentes 'naturales' que reproducen el mundo como lo conocemos y componentes innaturales que se mueven más allá de nuestro conocimiento del mundo real" (Alber, Nielsen y Richardson, 2012, p. 352). No obstante, a pesar de esta narratividad innatural ceñida a tal superconductividad, $L P C$ carece de una mayor elaboración del contexto social de la novela que parece coincidir (casi completamente) con el contexto de escritura de los autores de la misma, como ya se mencionó.

Con respecto a las intercalaciones, es claro que estas buscan crear un efecto estocástico, caótico, en el proceso de lectura. Este caos lectural tiene un fuerte vínculo con la idea misma de la superconductividad y el trasfondo mecánico-cuántico de esta última. La narración fragmenta, distrae y produce ambigüedad (como señalábamos arriba) para alejar al lector del relato del tiempo de investigación, con un caos lectural que plantea una dialéctica caos/orden, en cuanto es el lector quien escoge entre las diferentes posibilidades de lectura de las intercalaciones dadas y el orden en que serán estas asumidas. Mas, el orden está presente, en tanto las intercalaciones están señaladas intratextualmente en una secuencia (por escogencia) específica y que funciona dentro de un número limitado y definido de opciones (Sur, Oeste, Este, Norte y Cenit/Nadir), como jugando a las posibilidades con el gato de Schröndinger.

Asimismo, estas intercalaciones, que ya con el caos lectural establecido se definían como una narrativa innatural, también conllevan otro elemento de la narración innatural, donde "[l]as narrativas innaturales pueden ser vistas como lectores aludientes (cueingreaders) para engranarse en actividades decodificantes..." (Alber, Nielsen y Richardson, 2012, p. 364), que nos remiten nuevamente a la metaficcionalidad de estas narrativas postmodernas. Las intercalaciones incorporan, consecuentemente, otro elemento de las narrativas innaturales, o sea, "el establecimiento de la lectura y la escritura como 
colaboración" (Berry, 2012, p. 139), al querer incorporar al lector, mediante la elección del orden de lectura de las intercalaciones, en el proceso de (re)escritura.

Con todo esto, en $L P C$, se encuentra un dominante epistemológico en choque un dominante ontológico, que a nivel ideológico establece una tensión entre el énfasis mimético de las poéticas modernistas del dominante epistemológico y la propuesta de las narrativas innaturales con el énfasis diegético-metaficcional de las poéticas postmodernistas, lo cual deja a $L P C$ como una literatura experimental, no libre de contradicciones, donde se da “(...) el desmantelamiento de los valores clásicos incluso mientras permite un nuevo conjunto de valores, para establecer ellos mismos, sin reconocimiento y sin ningún escrutinio crítico, la celebración de la fragmentación sin la búsqueda acompañante de coherencia" (Olkowski, 2012, p. 4). En este sentido, LPC se estructura sobre una serie de valores modernos (como las nociones jurídicas que se aplican en el texto, como fundamento del proceso investigativo), pero que se fragmentan, no sin algún grado de incoherencia, para sostener valores postmodernos (como la ontología alterna, que sustenta el elemento ficto-científico de $L P C$ ). La mezcla de valores modernos y postmodernos, con la utilización de mecanismos narratológicos de poéticas tanto modernas como postmodernas, no permiten al texto un planteamiento profundo y sistemático de algunos problemas planteados en este (por ejemplo, la cuestión de la implícita crítica de lo jurídico en relación con las empresas transnacionales).

\section{Conclusión}

$L P C$ se define por el cruce onto-epistemológico de algunos elementos pertenecientes a las poéticas modernistas (el dominante epistemológico de la noción clásica del género detectivesco) y algunos elementos asimilados por la postmodernidad (como la teoría de la complejidad y algunas interpretaciones filosóficas de la mecánica cuántica, así como su relación con las narrativas innaturales), en el marco del desarrollo de un factor fictocientífico, como lo es la elaboración de superconductores a temperatura ambiente.

Dentro del contexto de la literatura costarricense, $L P C$ ejemplifica la producción de una literatura experimental en nuestro país, como un medio de explorar temáticas novedosas (como la teoría de la complejidad, la mecánica cuántica, la superconductividad, etc.) en el ámbito de concepciones narrativo-innaturales, que permiten la asunción de elementos narratológicos anclados en propuestas postmodernistas, que, en el espacio de la literatura costarricense, se podrían asimilar dentro de lo que Quesada (2000) denominaba la Promoción de 1980. Es decir, $L P C$ vendría a ser una manifestación tardía de esta promoción.

Como un primer acercamiento a $L P C$, se han establecido aquí algunos parámetros pertinentes y necesarios para el análisis de esta novela. Sin embargo, quedan muchas cuestiones por abordar, como: los vínculos existen de manera directa y específica entre esta novela y la Promoción de 1980 y la función metaléptica de las intercalaciones y otros elementos metaficcionales aquí señalados. 


\section{ABSTRACT}

\section{Los peces de Cooper (Cooper's Fish) and the Detective Genre: from the Epistemological Dominant to the Ontological Dominant}

This article analyses Los peces de Cooper from the classical to the metaphysical detective genre. The first one is in relation to modern poetic and the former to postmodern poetic. The analysis suggests a disagreement between the two poetics, through the incorporation of the fictional and scientific elements of the superconductivity. Likewise, this novel (Los peces de Cooper) is interpreted as an experimental piece of literature cling to the unnatural narratives.

Key words: Costa Rican literature, detective genre, physics, unnatural narrative, experimental literature.

\section{RÉSUMÉ}

\section{Los peces de Cooper (Les poissons de Cooper) et le genre policier: du dominant épistémologique au dominant ontologique}

Cet article analyse Los peces de Cooper en relation avec les paramètres du roman policier classique et du roman policier métaphysique; le premier est lié aux poétiques modernistes et le deuxième aux postmodernistes. L'analyse soulève le conflit entre les deux poétiques, à travers l'intégration de l'élément fictif-scientifique de la supraconductivité. De cette manière, ce roman (Los peces de Cooper) est interprété comme une littérature expérimentale ancrée dans les narrations non naturelles.

Mots-clés: littérature costaricienne, genre policier, physique, narrations non naturelles, littérature expérimentale.

\section{Bibliografía}

Alber, J.; Nielsen, H. S.y Richardson, B. (2012). Unnatural Voices, Minds, and Narration. The Routledge Companion to Experimental Literature. (pp. 351-367). London and New York: Routledge.

Alber, J.; Iversen, S.;Nielsen, H. S. y Richardson, B. (2013). What Really Is Unnatural Narratology? StoryWorlds: A Journal of Narrative Studies. 5, 101-118.

Berry, R. M. (2012). Metafiction. The Routledge Companion to Experimental Literature. (pp. 128-140). London and New York: Routledge.

Blundell, S. (2009). Superconductivity: A Very Short Introduction. New York: Oxford University Press.

Castellani, B. y Hafferty, F. W. (2009). Sociology and Complexity Science. A New Field of Inquiry. Heidelberg: Springer.

Cawelti, J. G. (1997). Canonization, Modern Literature, and the Detective Story. Theory and Practice of Classic Detective Fiction. (pp. 5-15). Westport, Connecticut y London: Greenwood.

Marcus, L. (2003). Detection and Literary Fiction. The Cambridge Companion to Crime Fiction. (pp. 245-267). New York: Cambridge University Press.

McHale, B. (1987). Postmodernist Fiction. London and New York: Routledge. 
McHale, B. (2012). Postmodernism and Experiment. The Routledge Companion to Experimental Literature. (pp. 141-153). London and New York: Routledge.

Merivale, P. (2010). Postmodern and Metaphysical Detection. A Companion to Crime Fiction. (pp. 308-320). Malden (MA) and Oxford: Wiley-Blackwell.

Nicol, B. (2009). The Cambridge Introduction to Postmodern Fiction. New York: Cambridge University Press.

Olkowski, D. E. (2012). Postmodern Philosophy and the Scientific Turn. Bloomington and Indianapolis: Indiana UniversityPress.

Ortega-Rodríguez, M. y Víquez-Jiménez, A. (2015). Los peces de Cooper. San José: EUNED.

Pyrhönen, H. (2010). Criticism and Theory. A Companion to Crime Fiction. (pp. 43-56). Malden (MA) and Oxford: Wiley-Blackwell.

Quesada Soto, Á. (2000). La narrativa costarricense del último tercio de siglo. Letras, Vol. XXXII, 17-43.

Ryan, Marie-Laure. (2012). Impossible Worlds. The Routledge Companion to Experimental Literature. (pp. 368-379). London and New York: Routledge.

Víquez Jiménez, A. (2014). Heisenberg y Bohr en la mira de Frayn y Volpi. Revista de Filología y Lingüística, Vol. 40 (2), 69-93.

Žižek, S. (2014). Event. A Philosophical Journey through a Concept. Brooklyn and London: Melville House. 
